\title{
A pair of gigantic bipolar dust jets close to the solar system
}

\author{
R. Weinberger and B. Armsdorfer
}

Institut für Astrophysik, Universität Innsbruck, Technikerstrasse 25, 6020 Innsbruck, Austria

e-mail: ronald.weinberger@uibk.ac .at; birgit.armsdorfer@uibk . ac . at

Received 4 December 2003 / Accepted 10 February 2004

\begin{abstract}
We present two adjacent jet candidates with a length of $\sim 9^{\circ}$ each $-10 \times$ longer than the largest known jets - discovered by us on $60 \mu \mathrm{m}$ and $100 \mu \mathrm{m}$ IRAS maps, but not observed at any other wavelength. They are extremely collimated (lengthto-width ratios 20-50), curved, knotty, and end in prominent bubbles. Their dust temperatures are $25 \pm 3 \mathrm{~K}$ and $30 \pm 4 \mathrm{~K}$, respectively. Both harbour faint stars, one having a high proper motion $\left(0.23 \mathrm{yr}^{-1}\right)$ and being very red, suggesting a distance of $\sim 60 \mathrm{pc}$. At this distance, the combined mass of both jets (assuming a gas-to-dust ratio of 200) totals $\sim 1 M_{\odot}$. We suspect that these gigantic ( $\sim 9$ pc length) jets have a common origin, due to the decay of a system of evolved stars. They are the first examples of jets radiating in the far IR and might be the closest non-diffuse nebulae to the solar system.
\end{abstract}

Key words. infrared: ISM, continuum - ISM: jets and outflows

\section{Introduction}

Collimated jets are one of the most fascinating but poorly understood phenomena in astronomy. They represent ubiquitous features and are found in quasars, active galactic nuclei, young stellar objects, symbiotic systems, planetary nebulae and pulsars. Their acceleration and collimation mechanisms might be the same in all the classes of objects (Livio 1999; Price et al. 2003). Although an agreement on the processes that drive all these jets has not yet been achieved, there is mounting evidence that bipolar ejection is powered by accretion and that magnetic fields play a crucial role in accelerating and collimating the gas (Anderson et al. 2003). The exact launching mechanism remains to be identified (Cabrit et al. 1999). The main reason is the rather large distance of even the nearest Galactic jets which preclude detailed observations of regions close enough to the star $(<$ several AU) where the jets are generated. The distances $(\gg 100 \mathrm{pc})$ also impede measurements of various important features like the cross-section structure of jets, internal knots and other microstructures. In this paper we present preliminary results on a pair of jets which obviously is unique in several respects and appears to be close to the solar system.

\section{Description of the two adjacent jets}

During a survey for large dust structures around planetary nebulae and white dwarfs on IRAS $12-100 \mu \mathrm{m}$ SkyView maps we detected two new bipolar jet candidates at high latitude $\left(b \approx+67^{\circ}\right)$ at 60 and $100 \mu \mathrm{m}$. The jets (objects A, B) are shown in Fig. 1. Object A (henceforth "A"; coined as "Giant Dumbbell

Send offprint requests to: $\mathrm{R}$. Weinberger, e-mail: ronald.weinberger@uibk.ac .at
Nebula" by us) consists of two roundish lobes (A1, A2) with a diameter of $\sim 1^{\circ} .2$. They have a central brightness depression $(\varnothing \sim 0.2)$ in $\mathrm{A} 2$ and are connected by a wavy (amplitude $\varnothing \sim 0.25)$, narrow $(\varnothing \sim 0.3)$ bridge of material with a length of 6.2 , leading to a length-to-width ratio of 21 . The total dimension of $\mathrm{A}$, whose long axis (A1-A2) has a position angle $\mathrm{PA}=83^{\circ} \pm 3^{\circ}$, is $8^{\circ}$. 6 . Its morphology reminds of Herbig-Haro (HH) jets produced by young stellar objects, except that bowshock nebulae are frequently seen at the ends of $\mathrm{HH}$ jets instead of the bubble-like structures in A. In SkyView we found no counterpart at other wavelengths except the "H $\alpha$ Composite All-Sky Survey", where an emission knot appears to coincide with A1 and a protrusion out of an extended emission structure appears to coincide with A2.

In Fig. 2, A is presented at $100 \mu \mathrm{m}$. The white line follows the wiggles in the jet, showing point symmetry with respect to its centre (white square). The round terminating bubbles A2 and A1 display a knotty appearance.

Object B (henceforth "B"; coined as "Wheat Sprout Nebula") consists of a knotty lobe $(\varnothing \sim 1$. 2 ), B1, connected to a faint thin curved bridge (length $\sim 11^{\circ}$ ) from B1's northern end to arc $\mathrm{b}^{\prime}$. The latter, of length $\sim 1.8$, is followed by a $\sim 0^{\circ} .7$ gap in emission and subsequently by arc $b^{\prime \prime}$ which is of length $\sim 1$. 7 . Farther to the north there is a faint possible counterpart to B1, named B2; $b^{\prime}+b^{\prime \prime}$ exactly match a $60^{\circ}$ long arc of a circle with curvature radius 3.8 . The centre of symmetry of $B$ is in the gap between $b^{\prime}$ and $b^{\prime \prime}$ and there is a clear reflection symmetry of the parts of $\mathrm{B}$ with respect to this centre. The total dimension of $\mathrm{B}$, whose long axis (B1-B2) has a $\mathrm{PA}=4^{\circ} \pm 1^{\circ}$, is $\sim 9^{\circ} .6$. By improving the resolution of IRAS images from $\sim 5^{\prime}$ to $\sim 1^{\prime}$ with HIRES, the knotty nature of $b^{\prime}$ and $b^{\prime \prime}$ becomes evident (Fig. 2). The five knots along $b^{\prime \prime}$ are spaced by $\sim 20^{\prime}$. The smooth 


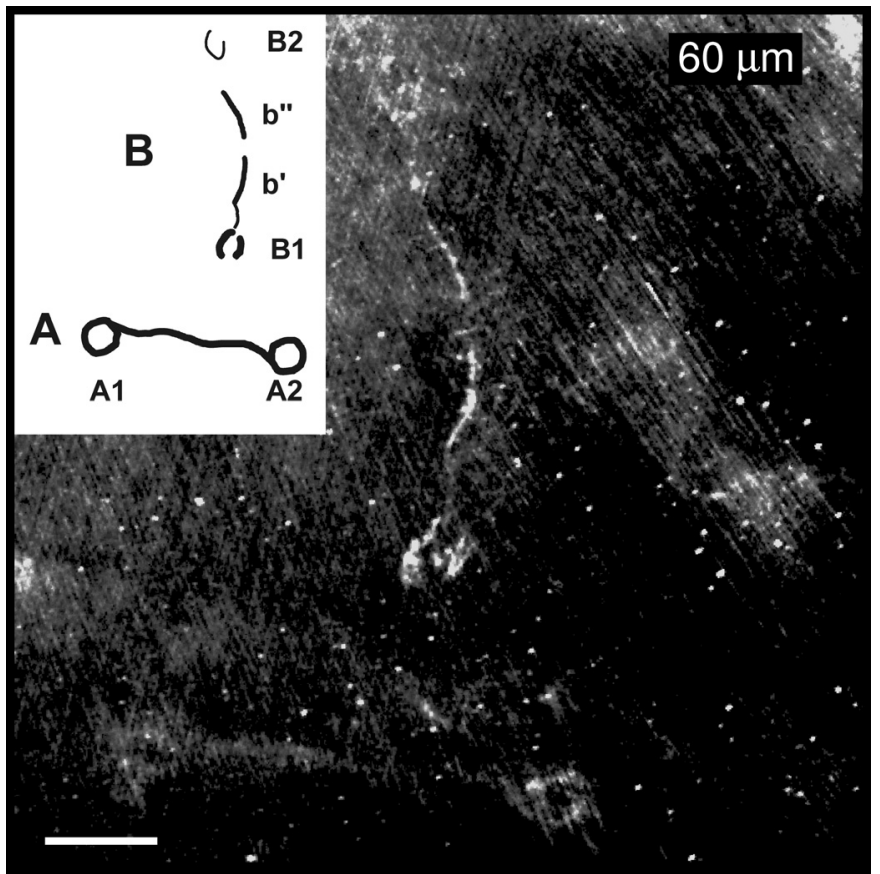

Fig. 1. A $15^{\circ} \times 15^{\circ}$ field centered at RA $=14^{\mathrm{h}} 23^{\mathrm{m}} 40^{\mathrm{s}}$, Dec $=$ $+40^{\circ} 00^{\prime} 00^{\prime \prime}(\mathrm{J} 2000)$ in the IRAS $60 \mu \mathrm{m}$ passband showing both bipolar jets. The sketch at upper left outlines the main features of the jets which are referred to in the text. The bar at lower left is $2^{\circ}$ long. North is at top, east to the left.

southern part of $b^{\prime}$ is of uniform width $\left(\sim 4^{\prime}\right)$, leading to an extreme length-to-width ratio of 52 for $b^{\prime}+b^{\prime \prime}$. HIRES contours show the knots to be mostly double, displaying steeper gradients in about upstream and downstream directions. The knots remind of internal working surfaces in $\mathrm{HH}$ jets, caused by varying jet ejection velocities. (On Jan. 28/29 2004 S. Temporin kindly obtained spectra with the $182 \mathrm{~cm}$ tel. of Asiago Observatory, at the position of the largest knot of $b^{\prime}$ and of the nebula $\sim 0$.9 east of jet A's center. With regard to the [SII] $671.6+673.1 \mathrm{~nm}$ lines, we found at best traces of emission in the knot, but very faint emission at the nebula's position.) See Eislöffel et al. (2000) and Reipurth \& Bally (2001) for reviews of $\mathrm{HH}$ objects.

In brief, A and B share the morphological characteristics with other classes of bipolar jets, but are unique in that they are emitting in the far IR. Their vicinity on the sky and their comparable length, shape and brightness suggest a common origin. The sources responsible for the ejection can hardly be young objects, since here we deal with a very transparent Galactic region, the Bootes Deep Field (which happens to coincide with the centre of $\mathrm{A}$ ) and there is no star forming region within at least $15^{\circ}$.

\section{Discussion}

We searched $\sim 1.5$ sqdeg around each jet centre (but also within B1) for possible ejecting sources on POSS I and POSS II. We found one stellar candidate for each jet. In A's very centre one faint red star with a high proper motion (p.m.) was detected (Fig. 3). We determined p.m. $=0$. $^{\prime} 23 \mathrm{yr}^{-1}$ along PA $=168^{\circ} \pm 3^{\circ}$,
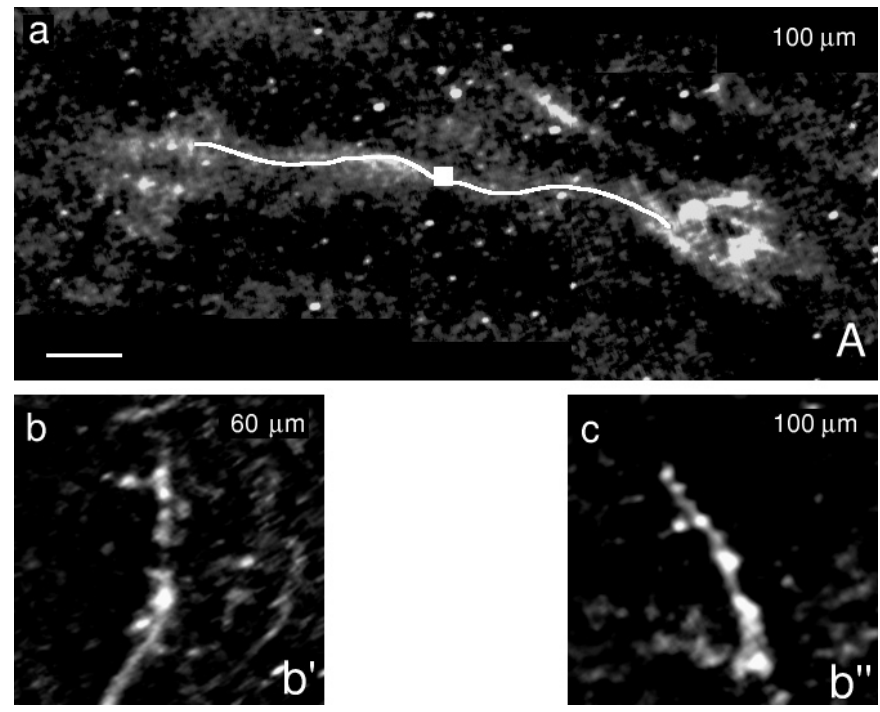

Fig. 2. Enlargements of object A and of two arcs of object B. The upper panel a) shows the southern jet at $100 \mu \mathrm{m}$, using images from the IRAS server in Groningen. At $100 \mu \mathrm{m}$ there is a higher background contamination compared to $60 \mu \mathrm{m}$, but the curved jet structure (point symmetric with respect to the white square plane) is better visible. The square $\left(\mathrm{RA}=14^{\mathrm{h}} 31^{\mathrm{m}} 19^{\mathrm{s}}\right.$, Dec $\left.=+34^{\circ} 14^{\prime} \cdot 2 ; \mathrm{J} 2000\right)$ marks the position of the central star candidate; the bar is $1^{\circ}$ long. In the lower left panel b) and lower right panel c) parts of $\mathbf{b}^{\prime}(60 \mu \mathrm{m})$ and $\mathbf{b}^{\prime \prime}(100 \mu \mathrm{m})$ of object B are shown respectively, processed with the HIRES (IRAS) tool. Both teem with knots. Note e.g., at part b's northern rim, the slightly curved horizontal emission streak, whose location suggests a possible relation with the jet. Both lower panels are $2^{\circ} \times 2^{\circ}$. North is at top, east to the left.

and a brightness $B=20.3 \pm 0.5, R=18.0 \pm 0.5$. In the 2MASS data archive we found $J=14.960 \pm 0.039, H=14.508 \pm 0.045$, and $K=14.166 \pm 0.047$. To estimate a distance $(D)$, we note that any $D>100 \mathrm{pc}$ leads to unreasonably high masses (and sizes) of the jets and in addition to an uncomfortably high velocity of the star - see below. In colour-colour diagrams, e.g. $(J-H)-(H-K)$, or colour-magnitude diagrams $\left(M_{J}-(J-K)\right.$, $M_{J}-(R-J)$, etc.) (Reid 2002) the star appears too blue for $D<100$ pc. Correcting for a possible weak blue contribution (companion or accretion disk?) by $0.1-0.2 \mathrm{mag}$ in $(J-K)$ shifts the star into the domain of late M dwarfs with $M_{J} \leq 11.5$ (Marley 2002). If the corrected $J$ is e.g. 0.3 mag fainter (15.26) and $M_{J}=11.3, D \sim 60 \mathrm{pc}$ follows. We will adopt this distance.

In $B$ we searched both within the area of B1 and in the assumed jet centre, i.e. between the arcs $b^{\prime}$ and $b^{\prime \prime}$. In the projected centre of B1, we located the eclipsing binary ROTSE1 J142344.96+374743.9. This system (GSC 2.2 mag: red $\sim 12.5$, blue $\sim 13.5$ ) however is several hundreds of pc away. In the centre of jet $\mathrm{B}$, no high proper motion star could be detected, but one faint bluish $\operatorname{star}(B=20.2 \pm 0.5, R=19.6 \pm 0.5$; $\mathrm{RA}=14^{\mathrm{h}} 21^{\mathrm{m}} 14.1$, Dec $=+41^{\circ} 54^{\prime} 53^{\prime \prime}(\mathrm{J} 2000)$ attracted our attention; it is only $\sim 8^{\prime}$ east of the gap's centre between $b^{\prime}$ and $b^{\prime \prime}$ and shows a faint $\left(\sim 1.6 \times 10^{-3} \mathrm{ct} / \mathrm{s}\right)$ ROSAT hri X-ray source a few arcsec north and south of it (perhaps even representing one single source coinciding with the star) (Fig. 3).

For the following discussion we assume that i) the 60 and $100 \mu \mathrm{m}$ emission is thermal emission from dust, ii) dust traces 

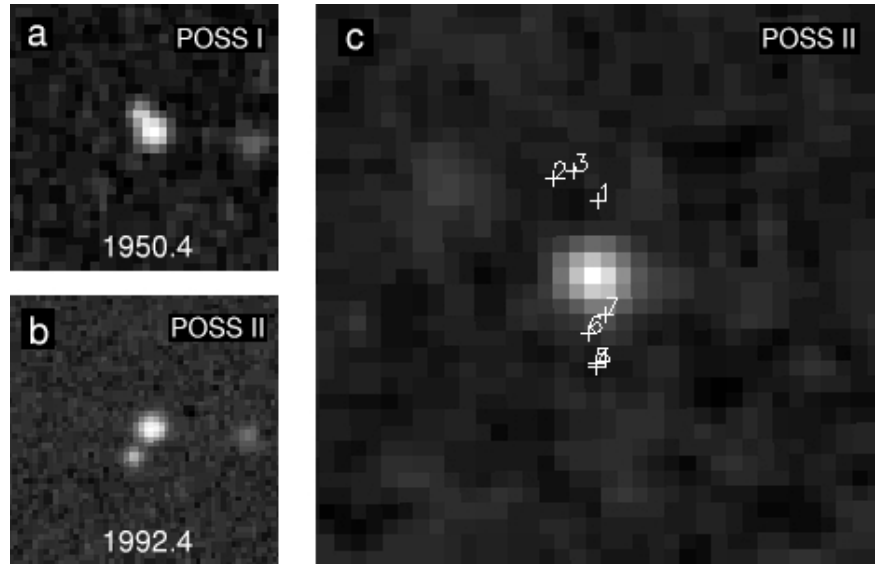

Fig. 3. The candidate ejection sources for the two jets. The left panels a), b) show the faint high-proper-motion star located in the centre of A (white square in Fig. 2), on plates of POSS I and POSS II, respectively. The brighter of the two stars probably is a background object. Both panels are $54^{\prime \prime} \times 54^{\prime \prime}$ wide. In the right panel (POSS II) the faint star in the centre of B (i.e. between arcs $b^{\prime}$ and $b^{\prime \prime}$ ) is presented, together with two groups of faint ROSAT hri X-ray sources (nos 1-7) according to the "ROSAT Complete Results Archive Sources for the HRI". However, these sources rather represent one source $\mathrm{N}$ and one $\mathrm{S}$ of the star, possibly even being only one single source. This panel is $36^{\prime \prime} \times 36^{\prime \prime}$ wide. For all images the DSS was used. Throughout, north is top and east to the left.

gas, and iii) the jets are in the plane of the sky. We obtained dust colour temperatures $T=25 \pm 3 \mathrm{~K}$ for $\mathrm{A}$ and $30 \pm 4 \mathrm{~K}$ for B. For $D=60 \mathrm{pc}$, with a gas-to-dust ratio of 200, and using the dust mass relation by Hildebrand (1983), we derived total masses of $0.99 \pm 0.67 M_{\odot}$ for A and $0.12 \pm 0.08 M_{\odot}$ for B. (At $D=100$ pc A's mass would be $2.8 M_{\odot}$ ). These low temperatures - similar to interstellar cirrus temperatures - and the lack of temperature gradients along the jets suggest that dust heating is mainly from the outside, probably from the interstellar radiation field. Hence, the jets might be cooled down fossils. The "fragmentation" of A1, A2 and B1, probably resulting from instabilities, backs this view. Interestingly, an effective collimation preventing the dust from expanding must still be at work (and should be addressed in future studies). This mechanism - we suspect magnetic fields - however appears to allow an abrupt widening of the jets at their end into round lobes which might actually be hollow spheres, unlike the usual bow-shocks in $\mathrm{HH}$ jets. We note that at $D=60 \mathrm{pc}$ we are inside the Local Bubble (LB), where the sound speed is high $\left(\gtrsim 100 \mathrm{~km} \mathrm{~s}^{-1}\right.$ ) and bow shocks may not form provided that the jet velocities are small enough.

We may estimate these jet velocities. Our pair probably has a common origin as suggested above. This is strongly supported by the p.m. vector of A's star, $168^{\circ} \pm 3^{\circ}$, which must be of low mass $\left(\gtrsim 0.1 M_{\odot}\right)$. If projected back, this star exactly arrives at the centre of B. In this centre we suspect the origin of the jet pair: we assume that there a triple or higher order stellar system had decayed. The member with the lowest mass, least bound to the system, has - together with its acquired accretion disk - been ejected along the plane of the sky and now represents the high p.m. star in the very centre of A. Jet A would be bent, with A's star at its apex (i.e. in the sense of a bow shock) only, if the jet has on its way suffered from noticeable interaction with ambient matter. Obviously this was not the case - as expected for a path within the LB.

Provided that the ejection of both jets started at the time when the stellar system broke up, then to cover the angular length of $8^{\circ} .1$ between the centre of B to the centre of $\mathrm{A}$ with $0.23 \mathrm{yr}^{-1}$ requires 127 kyears at $D=60 \mathrm{pc}$. The velocity of the star in the sky plane in this case amounts to $65 \mathrm{~km} \mathrm{~s}^{-1}$ (but would amount to, say, $\sim 220 \mathrm{~km} \mathrm{~s}^{-1}$ if the jets would be $D=200$ pc distant). Since A and B have total physical lengths of $9.6 \mathrm{pc}$ and $9.0 \mathrm{pc}$ respectively, the jet velocities (if constant) are $38 \mathrm{~km} \mathrm{~s}^{-1}$ and $36 \mathrm{~km} \mathrm{~s}^{-1}$. If these low values reflect the true conditions, then this explains why the jets' dust can survive. Further this is a hint to low mass stars as ejecting sources, since jet velocities are of the order of the escape velocities ${ }^{1}$.

The small p.m. of the possible source of B $(>10 \times$ smaller compared to the p.m. of the star in A) might imply a mass of $\geq 1 M_{\odot}$, in contradiction to the suggested low ejection velocity. Interestingly, the geometrically almost perfect reflection symmetry of jet B (above all, of the parts $b^{\prime}$ and $b^{\prime \prime}$ ) suggests an orbital motion of the ejecting source around a more massive object (Masciadri \& Raga 2002). (The point symmetry in A can according to these authors likewise be taken as an indication for precession; note, that a companion might be necessary for the precession, but a radiation-induced precession might work too.)

Anyway, we could not identify this object - the possible mass donor - and speculate that it might be of compact nature and could perhaps even be unresolvable from the bluish star at the scale of the POSS. If our suggestion is correct, then the material of the jets must originally have been copiously shed via a wind by a massive star (this would again speak in favour of a quite small $D$ given $b \approx+67^{\circ}$ ) and accreted by two low-mass members of the stellar system. The massive star could then, $>10^{5}$ years ago, have exploded as a supernova. Did this event contribute to the shape and hot gas of the LB? A search for a fossil wind cavity or supernova remnant led to a possible candidate: we used SkyView, selected a $50^{\circ} \times 50^{\circ}$ field, centered at $\mathrm{RA}=13^{\mathrm{h}} 55^{\mathrm{m}}$, Dec $=+35^{\circ}(\mathrm{J} 2000)$, and used "hist. equal." as brightness scaling. At $100 \mu \mathrm{m}$ (but not at $60 \mu \mathrm{m}$ ) a huge ellipsoidal shell-like structure, $\sim 40^{\circ} \times \sim 25^{\circ}$, with its long axis at PA $\sim 65^{\circ}$ becomes visible (Fig. 4). Its eastern rim is faintly present also in a corresponding $\mathrm{nH}$ radio map. The jet pair is located in the eastern part of this structure. The off-centre position might be due to asymmetric mass loss of the former massive star.

Alternatively, an AGB star might have been the mass donor. This star should however be still present as a bright (at least $10^{2}-10^{3} L_{\odot}$ ) blue object. If the faint bluish star close to the centre of B represents this young white dwarf, it must however be heavily obscured by the (dust) accretion disk. Indeed, we

1 The referee made the following comment: if one star supplies the mass to the accretion disk of each of the two stars, one blew A and one blew B, then one of them (that of A) left the massive star quite early. Accretion disks fade quite fast - few times the dynamical time after no mass is supplied. Therefore, it is quite possible that jet A was blown for a very short time only. 


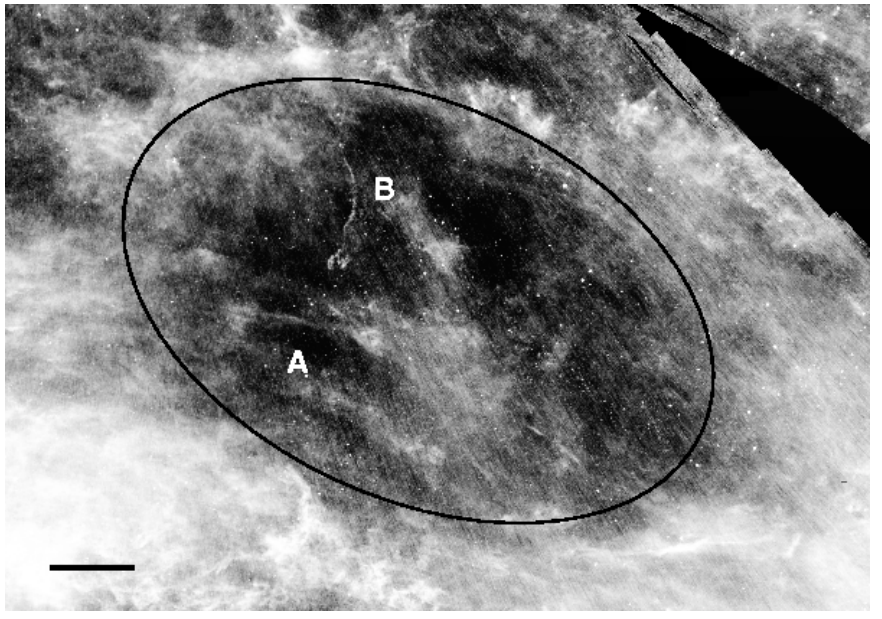

Fig. 4. A $50^{\circ} \times 35^{\circ}$ field centered at RA $=13^{\mathrm{h}} 55^{\mathrm{m}}$, Dec $=+35^{\circ}$ (J2000), i.e. $1, b=67^{\circ},+74^{\circ}$, in the IRAS $100 \mu \mathrm{m}$ passband showing a huge ellipsoidal shell. Objects A and B are marked. The bar is $5^{\circ}$ long. North is at top, east to the left.

expect a more or less edge-on disk in both $\mathrm{A}$ and $\mathrm{B}$, but the bluish nature of the candidate ejection source in $\mathrm{B}$ implies that a blue excess has to be present. Since near to this star two (or one) X-ray source(s) appear to exist (Fig. 3), such an excess could be explained. An other problem with the AGB star scenario is, that much less mass is lost (and consequently accreted) than in the former case and that a decay of a stellar system is less probable. A solution of this problem has to wait for detailed observations of the presently known candidates.

\section{Conclusion}

Two gigantic adjacent jet candidates, found in the far infrared, have been discovered. They represent the first examples of jets radiating in this wavelength range. Due to their vicinity on the sky and their comparable length, shape, high degree of collimation and brightness, and a proper motion of one of the candidate ejecting stars which obviously links both jets, we suggest a common origin. The sources responsible for the ejection can hardly be young objects, since the jet pair is far away from any star forming region. The physical size of the jets and the candidate ejecting star of one of the jets with its very high proper motion suggest a close distance, less than $100 \mathrm{pc}$, i.e. within the LB. No clear conclusion can be drawn on the origin of the jets, but we suggest that they stem from a decayed stellar system, where a massive star was a mass donor and has generated accretion disks around two low-mass stellar members. The jet pair appears to contain cooled-down material, i.e. it is of fossil type. Other fossil jets may exist, but will not be easily detectable because of the ubiquitous presence of interstellar dust.

A wealth of observations will be necessary to unravel details of these enigmatic adjacent jets. Primary goals should be to carry out high-resolution imaging and spectroscopic observations of the candidate ejection sources, and optical spectroscopy and $\mathrm{CO}$ observations of several portions of the jets. First and foremost, the derivation of a reliable distance should be undertaken. Apart from understanding its very nature, this pair might be important for future studies of the acceleration and collimation processes in astrophysical jets due to its closeness.

Acknowledgements. Part of this work was supported by the Austrian Fonds zur Förderung der wissenschaftlichen Forschung (FWF), project number P15316. We thank M. V. van der Sluys for fruitful discussions, comments and criticism. Further we are much obliged to S. Temporin for taking and discussing optical spectra of parts of jet $A$ and jet B at the Asiago Observatory. We also thank B. Aryal for his help in handling IRAS maps and S. Kimeswenger for various comments.

\section{References}

Anderson, J. M., Li, Z.-Y., Krasnopolsky, R., \& Blandford, R. D. 2003, ApJ, 590, L107

Cabrit, S., Ferreira, J., \& Raga, A. A. 1999, A\&A, 343, L61

Eislöffel, J., Mundt, R., Ray, T. P., \& Rodriguez, L. F. 2000, in Protostars and Planets IV, Collimation and Propagation of Stellar Jets, ed. V. Mannings, A. P. Boss, \& S. S. Russell (Tucson: Univ. of Arizona Press), 815

Hildebrand, R. H. 1983, Q. Jl. R. Astr. Soc., 24, 267

Livio, M. 1999, Phys. Rep., 311, 225

Marley, M. S., Ackerman, A., Burgasser, A. J., et al. 2002 [arXiv: astro-ph/0207658v1]

Masciadri, W., \& Raga, A. C. 2002, ApJ, 568, 733

Price, J., Pringle, J. E., \& King, A. R. 2003, MNRAS, 339, 1223

Reid, N. 2002, http://www-int. stsci. edu/ inr/cmd.html

Reipurth, B., \& Bally, J. 2001, ARA\&A, 39, 403 